\title{
Enfermedad inflamatoria crónica intestinal canina: hallazgos endoscópicos, bioquímicos y anatomopatológicos del tracto gastrointestinal anterior
}

\author{
Canine inflammatory bowel disease: endoscopic, biochemical and pathologic \\ findings in anterior gastrointestinal tract \\ R Crespo ${ }^{\mathrm{a}}$, P Cámara ${ }^{\mathrm{a}}$, A Buendía ${ }^{\mathrm{b}}$, I Ayala ${ }^{\mathrm{a}}$ \\ aDepartamento de Medicina y Cirugía Animal, Facultad de Veterinaria, Hospital Clínico Veterinario, \\ Campus de Espinardo, Murcia, España. \\ ${ }^{\text {b}}$ Departamento de Anatomía y Anatomía Patológica Comparadas, Facultad de Veterinaria, Hospital Clínico Veterinario, \\ Campus de Espinardo, Murcia, España.
}

\begin{abstract}
SUMMARY
A retrospective study was carried out in 26 dogs referred to the Internal Medicine Service (Endoscopy Unit) of the Clinical Veterinary Hospital of Murcia University, diagnosed with inflammatory bowel disease affecting the anterior gastrointestinal tract. Haematological, endoscopic and pathologic findings were considered to assess potential correlation between these parameters. Neither hypoproteinemia nor hypoalbuminemia had a significant correlation with the degree of inflammation in the gastrointestinal mucosa. However, the animals with a lymphoplasmacytic infiltrate, presented a tendency to hypoalbuminemia. A statistically significant correlation $(\mathrm{P}<0.05)$ was found between the type of infiltrate and the finding of congestion in gastroduodenal mucosa. An increased congestion was related to the lymphoplasmocytary infiltrate. There was also a correlation between the degree of congestion and the infiltrate intensity in the gastroduodenal mucosa, which approximated to the statistical significance, in spite of not being statistically significant $(\mathrm{P}<0.1)$. In addition, friability is associated with inflammation in duodenum, and the animals who did not have a proper closure of the cardia presented esophagitis by reflux.
\end{abstract}

Key words: inflammatory bowel disease, dog, correlation endoscopic-pathologic findings.

RESUMEN

Se ha realizado un estudio retrospectivo a 26 perros, machos y hembras, que acudieron al Servicio de Medicina Interna (Unidad de Endoscopia) del Hospital Clínico Veterinario de la Facultad de Veterinaria de la Universidad de Murcia (España), a los que se les diagnosticó enfermedad inflamatoria crónica intestinal del tracto gastrointestinal anterior. Los casos se eligieron en función del diagnóstico clínico, endoscópico y anatomopatológico realizado, con el objetivo de evaluar potenciales correlaciones entre estos parámetros. La disminución de proteínas totales y la albúmina no fue estadísticamente significativa en relación con el grado de inflamación de la mucosa gastrointestinal, no obstante, los animales que presentaban un infiltrado de tipo linfoplasmocitario tenían tendencia a la pérdida de albúmina. Se encontró una correlación estadísticamente significativa $(\mathrm{P}<0,05)$ entre el tipo de infiltrado y la presencia de congestión en la mucosa de estómago y duodeno. Una mayor congestión a estos niveles se corresponde con el infiltrado linfoplasmocitario. También se obtuvo un resultado que, a pesar de no ser estadísticamente significativo, sí se aproxima a la significación estadística $(\mathrm{P}<0,1)$ entre el grado de congestión y la intensidad del infiltrado, ya que un grado de congestión mayor de la mucosa se corresponde con una mayor intensidad de infiltrado linfoplasmocitario, tanto a nivel gástrico como duodenal. Además se vio que la friabilidad está asociada a inflamación en duodeno, y los animales que no tenían un correcto cierre del cardias presentaban esofagitis por reflujo.

Palabras clave: enfermedad inflamatoria intestinal, perro, correlación hallazgos endoscópicos-histológicos.

\section{INTRODUCCIÓN}

Los procesos digestivos constituyen una de las casuísticas que con mayor frecuencia encontramos en la clínica veterinaria de pequeños animales, aproximadamente $20 \%$ de los pacientes que entran a la consulta presentan algún desorden gastrointestinal (Simpson y Else 1991). Dentro de estos se encuentra la enfermedad inflamatoria crónica intestinal (EICI, IBD en inglés, siglas correspondientes a

Aceptado: 19.03.2015.

* iayape@um.es
Inflammatory Bowel Disease), la que se define como la presencia de signos gastrointestinales crónicos (más de tres semanas de duración), persistentes o recurrentes, lo que confiere su carácter crónico de curso impredecible, acompañados de evidencia histopatológica de inflamación de la mucosa, cuya etiología es indeterminada. Es un proceso idiopático, no obstante, la hipótesis principal es que puede tratarse de una respuesta agresiva, exagerada o inapropiada del sistema inmune a antígenos bacterianos o alimentarios (Hall y German 2007, Washabau y col 2010, Willard 2010). Esta teoría estaría apoyada por el incremento del número de células plasmáticas secretoras de inmunoglobulinas y la presencia de células $\mathrm{T}$ en 
tejidos inflamados (Washabau y col 2010). Los factores predisponentes serían de origen genético y condicionarían la regulación de la respuesta inmune y la permeabilidad intestinal. Estas predisposiciones genéticas se han visto en varias razas caninas como el Pastor Alemán, Basenji, Shar Pei y Soft-coated Wheaten Terrier (Washabau y col 2010). Suele presentarse en animales de edad media o avanzada. No responden a las terapias dietéticas, antiparasitarias y antibióticas, y sí hay respuesta clínica al uso de agentes antiinflamatorios o inmunosupresores. Los signos clínicos, como vómitos, diarrea del intestino delgado, diarrea del intestino grueso, pérdida de peso o alteraciones en el apetito, se deben a estas alteraciones inflamatorias e infiltrados celulares de la mucosa que dan lugar a una disfunción de los enterocitos, y por tanto a una dismotilidad intestinal (Washabau y col 2010). Es importante mencionar que por disfunción del esfínter gastroduodenal (píloro), debido al proceso inflamatorio, puede extenderse el proceso y provocar gastritis, siendo la manifestación clínica el vómito crónico, acompañado o no de diarrea (Hall y German 2007). El proceso inflamatorio se denomina sobre la base del tipo de infiltrado predominante presente en la lámina propia, entre los que se encuentran, por orden de frecuencia, enteritis linfoplasmocitaria, que se caracteriza por la presencia de linfocitos y células plasmáticas; enteritis eosinofílica, cuyo infiltrado está compuesto por eosinófilos, y enteritis neutrofílica, en caso que el infiltrado esté compuesto en su mayoría por neutrófilos. Pueden coexistir varios tipos inflamatorios en número similar, haciendo difícil su clasificación. Para llegar al diagnóstico definitivo se requiere el estudio histopatológico de las muestras obtenidas mediante biopsia (Washabau y col 2010). Generalmente se suele preferir la toma de biopsias por métodos mínimamente invasivos, como la endoscopia, por lo que este método diagnóstico se utiliza en la mayor parte de los casos para llegar al diagnóstico definitivo. En patología endoscópica digestiva, los hallazgos más frecuentes en animales con EICI son gastritis, duodenitis o colitis. Uno de los problemas que se encuentran al realizar una endoscopia es cuantificar en su justa medida los hallazgos macroscópicos que presenta la mucosa lesionada, de tal manera que no siempre es fácil establecer una relación entre lo que se observa y el grado de gravedad que realmente presenta el animal. En un estudio publicado por Day y col (2008) se hacía referencia a la ausencia de estandarización para definir las características morfológicas e inflamatorias de muestras tomadas endoscópicamente en perros y gatos con enfermedad gastrointestinal. No obstante, recientemente se ha logrado establecer una base de criterios comunes para clasificar los hallazgos histopatológicos de la EICI y esta estandarización es importante para la correcta evaluación de las muestras, y por tanto para un correcto diagnóstico y tratamiento aunque siguen existiendo dificultades acerca de una aceptación universal. Se sigue encontrando dificultad en el análisis objetivo de datos, como la distinción de un tejido normal de uno enfermo, la caracterización de la naturaleza y de la gravedad de la enfermedad, además de la variación en la interpretación de las muestras entre distintos anatomopatólogos (Day y col 2008). Además, debido a esta anterior falta de estandarización, no se han podido comparar resultados entre diversos autores. Pocos estudios en medicina veterinaria correlacionan de una forma detallada y exhaustiva los hallazgos endoscópicos con los histológicos, aunque sí existen en la literatura trabajos (Willard y col 2002, Craven y col 2004, GarcíaSancho y col 2007, Day y col 2008) que correlacionan los hallazgos clínicos con la gradación histológica de la EICI. Como se mencionaba en otro estudio publicado por Washabau y col (2010), no se ha identificado una sólida asociación entre hallazgos clínicos y lesiones histológicas en perros con EICI.

Por ello se plantea estudiar de forma retrospectiva varios casos con diagnóstico confirmado histopatológicamente de EICI a partir de muestras de biopsia obtenidas mediante endoscopia del tracto gastrointestinal anterior, en los que se ha valorado con una escala semicuantitativa diferentes parámetros endoscópicos, y además han sido analizadas con una escala similar los parámetros histológicos, a la vez que se trata de obtener información suplementaria mediante técnicas inmunohistoquímicas. El objetivo del presente estudio es correlacionar los hallazgos endoscópicos, bioquímicos e histológicos mediante estudio estadístico, de tal manera que obtengamos conclusiones con significación estadística para obtener una mejor valoración de la EICI canina en la exploración endoscópica.

\section{MATERIAL Y MÉTODOS}

\section{CRITERIO DE SELECCIÓN DE LOS CASOS CLÍNICOS, INCIDENCIA Y SINTOMATOLOGÍA CLÍNICA}

Los casos clínicos se seleccionaron a partir de consultas remitidas por otros centros veterinarios al Servicio de Medicina Interna (Unidad de Endoscopia) del Hospital Clínico Veterinario de la Facultad de Veterinaria de la Universidad de Murcia (España). Al ser un estudio retrospectivo, se seleccionaron para este trabajo de investigación 26 casos clínicos de varios años anteriores (2002-2005), con síntomas digestivos como vómitos, diarrea, pérdida de peso, y que presentaban como diagnóstico definitivo enfermedad inflamatoria crónica intestinal. Las terapias instauradas en un primer momento por los veterinarios remitentes no resolvieron el cuadro. El diagnóstico de estos pacientes se basó en la realización de endoscopia del tracto digestivo anterior (gastroduodenoscopia) y en el estudio histopatológico de las biopsias obtenidas de la mucosa gástrica y duodenal. Los casos clínicos eran relativos a 26 perros de diferentes razas, edades y sexo, y el tiempo que llevaban presentando dicha sintomatología antes de su diagnóstico definitivo oscilaba entre 10 y 35 semanas (cuadro 1). Estos parámetros referentes a la raza, edad, 
Cuadro 1. Detalle de la raza, edad, sexo, peso, síntoma principal y tiempo de padecimiento de la enfermedad de los perros incluidos en el presente estudio.

Details of the race, age, sex weight, main symptom and length of illness of the dogs included in the study.

\begin{tabular}{|c|c|c|c|c|c|c|}
\hline Caso & Raza & $\begin{array}{l}\text { Edad } \\
\text { (años) }\end{array}$ & Sexo & $\begin{array}{l}\text { Peso } \\
(\mathrm{kg})\end{array}$ & Síntoma principal & $\begin{array}{l}\text { Tiempo de enfermedad } \\
\text { (semanas) }\end{array}$ \\
\hline 1 & Fox-Terrier & 11 & Macho & 13 & Diarrea & 16 \\
\hline 2 & Caniche & 7 & Macho & 5 & Vómitos, diarrea & 10 \\
\hline 3 & Mestizo & 13 & Macho & 10 & Vómitos, diarrea & 15 \\
\hline 4 & Pastor Alemán & 3 & Macho & 23,3 & Diarrea & 17 \\
\hline 5 & Dálmata & 7 & Macho & 21 & Diarrea & 14 \\
\hline 6 & Westy & 3 & Hembra & 5,7 & Vómitos, diarrea & 12 \\
\hline 7 & Cocker & 4 & Macho & 9,3 & Diarrea, pérdida de peso & 27 \\
\hline 8 & Westy & 1,5 & Macho & 6,5 & Vómitos, diarrea & 14 \\
\hline 9 & Chihuahua & 4 & Macho & 1,7 & Vómitos, diarrea & 13 \\
\hline 10 & Mestizo & 2 & Macho & 3,4 & $\begin{array}{l}\text { Vómitos, diarrea, pérdida } \\
\text { de peso }\end{array}$ & 24 \\
\hline 11 & Caniche & 10 & Hembra & 7,5 & Diarrea, pérdida de peso & 32 \\
\hline 12 & Yorkshire Terrier & 2 & Hembra & 2,8 & Vómitos, diarrea & 14 \\
\hline 13 & Mestizo & 2 & Hembra & 7 & Vómitos, diarrea & 18 \\
\hline 14 & Beagle & 2 & Hembra & 12,4 & Vómitos, diarrea & 24 \\
\hline 15 & Yorkshire Terrier & 11 & Hembra & 4,5 & Vómitos, diarrea & 17 \\
\hline 16 & Schnauzer & 4,5 & Macho & 7 & Diarrea, pérdida de peso & 30 \\
\hline 17 & Mestizo & 8 & Macho & 13,5 & Vómitos, diarrea & 25 \\
\hline 18 & Mestizo & 8 & Macho & 5,4 & Diarrea & 16 \\
\hline 19 & Labrador Retriever & 9 & Macho & 28 & Diarrea, pérdida de peso & 28 \\
\hline 20 & Mestizo & 2,5 & Macho & 30 & $\begin{array}{l}\text { Vómitos, diarrea, pérdida } \\
\text { de peso }\end{array}$ & 35 \\
\hline 21 & Mestizo & 3 & Hembra & 5,5 & Vómitos, diarrea & 21 \\
\hline 22 & Pastor Alemán & 9 & Macho & 25,6 & Diarrea & 26 \\
\hline 23 & Rottweiler & 10 & Macho & 36 & Diarrea, pérdida de peso & 33 \\
\hline 24 & Bóxer & 8 & Macho & 26,3 & Diarrea & 18 \\
\hline 25 & Cocker & 7 & Hembra & 10,3 & Diarrea, pérdida de peso & 27 \\
\hline 26 & Bichón Maltés & 3 & Hembra & 3,1 & Vómitos, diarrea & 12 \\
\hline
\end{tabular}

sexo y sintomatología clínica también fueron analizados en el presente trabajo.

\section{PRUEBAS DIAGNÓSTICAS COMPLEMENTARIAS}

A todos estos pacientes se les realizó, antes de la exploración endoscópica, un análisis bacteriológico y parasitológico de las heces para descartar infecciones o parasitosis gastrointestinales, para lo que se procedió a la recogida de heces durante tres días consecutivos de cada uno de los animales incluidos en el estudio. Para el análisis bacteriológico se hizo un cultivo de las muestras fecales en medios artificiales, como Agar EMB o Agar Levine (medio selectivo para el aislamiento y diferenciación de E. coli), y Agar Salmonella Shigella (medio de cultivo utilizado para el aislamiento de Salmonella spp. y de algunas especies de Shigella spp.). Para el estudio parasitológico se utilizó el método Telemann modificado, una técnica de sedimentación para la detección de quistes de protozoos, huevos y larvas de helmintos. También se realizó analítica sanguínea (hematología y bioquímica clínica) para descartar alteraciones metabólicas; y Test de tripsina inmunorreactiva (TLI) en suero sanguíneo, mediante técnicas de radioinmunoanálisis, para descartar insuficiencia pancreática exocrina. Los parámetros bioquímicos analizados para comprobar el funcionamiento e integridad hepáticos fueron alaninoaminotransferasa (ALT), aspartato aminotransferasa (AST), gamma glutamil transferasa (gGT), fosfatasa alcalina y bilirrubina total, para chequear el funcionamiento renal se utilizaron creatinina y urea. Igualmente fueron determinadas las proteínas séricas totales y la albúmina. 


\section{TÉCNICA ENDOSCÓPICA UTILIZADA}

Para la realización de la gastroduodenoscopia de los animales se les mantuvo en ayunas las 24 horas previas al estudio, y 12 horas antes de la exploración se les retiró el agua. Como protocolo anestésico utilizado se premedicó a los animales con dexmedetomidina $(12 \mu \mathrm{g} / \mathrm{kg}$; Dexdomitor $0,5 \mathrm{mg} / \mathrm{ml} \circledast$, Zoetis) y butorfanol $\left(0,1 \mathrm{mg} / \mathrm{kg}\right.$; Torbugesic ${ }^{\circ}$, Fort Dodge Laboratories) por vía intramuscular, y aproximadamente media hora después, al obtener un grado de sedación adecuado, se cateterizó una vena cefálica con el catéter intravenoso del tamaño más apropiado para cada paciente. Se indujo la anestesia con propofol (PropofolLipuro® 1\%, B. Braun Medical S.A.) por vía intravenosa, a dosis efecto. Se procedió a la intubación endotraqueal, con tubos endotraquales de diámetro variable según el tamaño del animal. El mantenimiento anestésico se realizó con anestesia inhalatoria, concretamente isofluorano (Isoflo®, Esteve), a una concentración espirada en torno al 1,3\% administrando una mezcla de oxígeno al $40 \%$ y aire, hasta el final de la exploración endoscópica. Para la realización de la misma se empleó un videoendoscopio Fujinon ${ }^{\circledR}$ EG-410 HB (Saitama, Japón) flexible de $110 \mathrm{~cm}$ x $9 \mathrm{~mm}$ con capacidad de desvío de punta en cuatro direcciones y con un canal de trabajo de 2,5 mm para la introducción de pinzas de biopsias para la toma de muestras. Durante la endoscopia, el trayecto explorado fue esófago cervical y torácico, fundus y cuerpo gástrico, antro pilórico y la porción craneal del duodeno. Se tomaron de 6 a 10 muestras de biopsia de distintos puntos de la mucosa gástrica y duodenal.

\section{CRITERIO DE EVALUACIÓN DE LESIONES MACROSCÓPICAS OBSERVADAS EN ENDOSCOPIA}

Se sometió a los animales a una evaluación macroscópica de las lesiones encontradas en estómago y duodeno.
A todas ellas se les asignó un valor o puntuación en una escala semicuantitativa, como las realizadas por Washabau y col (2010) en función de los criterios del endoscopista, el que realizó todos los exámenes endoscópicos de los animales incluidos en este estudio, siendo el valor más bajo, 0 , el correspondiente a mucosa normal o no alterada y los valores más altos, de 1 a 3 , correspondientes gradualmente a una mayor gravedad en las lesiones (leve, moderado y grave). Los parámetros endoscópicos estudiados en el presente trabajo son aquellos relacionados con el proceso inflamatorio crónico que aparece en esta enfermedad, por lo que tanto en estómago como en duodeno se evaluó la presencia y gravedad de hemorragias, erosiones, úlceras, congestión, engrosamiento y granularidad de la mucosa; y en el duodeno se valoró además la friabilidad en el momento de coger las muestras de biopsia. Por lo demás, se evaluaron dos parámetros adicionales: el correcto cierre del cardias y la presencia o ausencia de esofagitis por reflujo (cuadro 2).

\section{CRITERIO DE EVALUACIÓN DE LAS LESIONES MICROSCÓPICAS DE LAS MUESTRAS DE BIOPSIA}

Se realizó el estudio histopatológico de las muestras de biopsia a partir de secciones incluidas en parafina de 5 $\mu \mathrm{m}$ de grosor teñidas mediante la técnica de HematoxilinaEosina. Las muestras fueron valoradas al microscopio óptico siguiendo las directrices del grupo de estandarización gastrointestinal de la World Small Animal Veterinary Association (WSAVA; Day y col 2008) para el diagnóstico de la inflamación gastrointestinal de biopsias endoscópicas en el perro y el gato. Estos parámetros, para nuestra zona de estudio (tracto gastrointestinal anterior), incluyen la valoración de rasgos morfológicos como acortamientoensanchamiento de vellosidades, lesión epitelial, dilatación de vasos linfáticos y fibrosis; los parámetros relacionados

Cuadro 2. Criterio de evaluación de lesiones macroscópicas observadas en endoscopia.

Evaluation criteria of the macroscopic lesions observed during endoscopy.

\begin{tabular}{|c|c|c|c|c|c|c|c|}
\hline & & $\begin{array}{l}\text { Estado } \\
\text { normal }\end{array}$ & $\begin{array}{l}\text { Afectación } \\
\text { leve }\end{array}$ & $\begin{array}{c}\text { Afectación } \\
\text { moderada }\end{array}$ & $\begin{array}{l}\text { Afectación } \\
\text { grave }\end{array}$ & Presencia & Ausencia \\
\hline \multirow{5}{*}{$\begin{array}{l}\text { Estómago y } \\
\text { duodeno }\end{array}$} & Hemorragia & 0 & 1 & 2 & 3 & & \\
\hline & Erosión & 0 & 1 & 2 & 3 & & \\
\hline & Úlcera & 0 & 1 & 2 & 3 & & \\
\hline & $\begin{array}{l}\text { Congestión de la } \\
\text { mucosa }\end{array}$ & 0 & 1 & 2 & 3 & & \\
\hline & $\begin{array}{l}\text { Engrosamiento e } \\
\text { irregularidad de la } \\
\text { mucosa }\end{array}$ & 0 & 1 & 2 & 3 & & \\
\hline Duodeno & Friabilidad & 0 & 1 & 2 & 3 & & \\
\hline \multirow{2}{*}{ Esófago } & $\begin{array}{l}\text { Cierre correcto del } \\
\text { cardias }\end{array}$ & & & & & 0 & 1 \\
\hline & Esofagitis por reflujo & & & & & 1 & 0 \\
\hline
\end{tabular}


con la inflamación incluyen la valoración de linfocitos intraepiteliales, infiltrado de linfocitos y células plasmáticas en lámina propia, infiltrado de eosinófilos en lámina propia e infiltrado de neutrófilos en lámina propia. Para cada uno de estos parámetros hay cuatro categorías, que son mucosa normal, lesión leve, moderada o grave.

\section{ESTUDIO INMUNOHISTOQUÍMICO}

Las técnicas inmunohistoquímicas permiten detectar una gran variedad de antígenos presentes en las células o tejidos, utilizando anticuerpos marcados, permitiendo de esta forma caracterizar los diferentes componentes del infiltrado inflamatorio. Estas técnicas se basan en la capacidad de los anticuerpos de unirse específicamente a sus antígenos correspondientes. Se llevó a cabo un estudio inmunohistoquímico para poner de manifiesto la proteína nuclear Ki-67, determinante del índice de proliferación celular entre las células del infiltrado inflamatorio; así como los marcadores de linfocitos CD3 (para linfocitos T) y CD79 (para linfocitos B), estos se utilizaron como complemento al diagnóstico de las lesiones encontradas histopatológicamente, el primero de ellos pone de manifiesto la presencia de linfocitos $T$, y el segundo se tiñe en caso de estar presentes linfocitos B; y también marcadores de MHC II, para identificar la presencia de antígenos mediante el complejo principal de histocompatibilidad. El estudio inmunohistoquímico se realizó sobre cortes de muestras de biopsias endoscópicas fijadas en formaldehído al $10 \%$ e incluidos en parafina, mediante la técnica del Complejo Avidina-Biotina-Peroxidasa (ABC) (Hsu y col 1981). Los elementos utilizados en este estudio procedieron del Laboratorio Dako, y las diluciones fueron 1:100 para Ki-67, 1:50 para CD3, 1:100 para CD79 y 1:50 para MHC II.

\section{ANÁLISIS ESTADÍSTICO}

Para evaluar la significación de los resultados se llevó a cabo un análisis estadístico, mediante el programa SPSS 15,0 realizando tablas de contingencia entre las variables, a partir de ellas analizamos las frecuencias de estas mediante un Estadístico exacto de Fisher. Para el análisis de la relación de variables expresadas se utilizó la técnica de Chi-cuadrado. Se consideró que, a pesar de no ser estadísticamente significativo, $\mathrm{P}<0,1$ sí se aproxima a la significación estadística; y una relación estadísticamente significativa cuando $\mathrm{P}<0,05$. Las variables seleccionadas para valorar la existencia de correlación estadística fueron la albúmina y el grado de inflamación de la mucosa gastrointestinal, la albúmina y tipo de infiltrado inflamatorio, el tipo de inflamación y la presencia de congestión endoscópica de la mucosa del tracto gastrointestinal anterior, el grado de congestión endoscópica y la intensidad del infiltrado inflamatorio, la friabilidad e inflamación del duodeno, y la presencia de esofagitis e incompetencia del cardias.

\section{RESULTADOS}

\section{INCIDENCIA DE LA PRESENTACIÓN DE LA ENFERMEDAD}

En cuanto al género, raza y edad, en este estudio estaban afectados animales tanto machos $(65,38 \%)$ como hembras $(34,62 \%)$ de diferentes razas, abarcando una amplia variedad de las mismas, siendo las de mayor representación en este estudio, por orden decreciente, mestizo, Caniche, Pastor Alemán, Westy, Cocker, y Yorkshire Terrier, y eran tanto jóvenes como de edad media y avanzada, siendo la edad media de presentación de la enfermedad de 5,94 años, con una desviación típica de 3,50.

\section{SINTOMATOLOGÍA CLÍNICA}

Los síntomas por los cuales los propietarios de los perros de este estudio acudieron a su clínica veterinaria fueron, en primer lugar, la presencia de diarrea, acompañada o seguida en ocasiones de otros síntomas como vómitos, y con las terapias instauradas en un primer momento, tanto dietéticas, como antibióticas o antiparasitarias, no se consiguió resolver el cuadro, o en algunos casos empeoraba y empezaban a presentar pérdida de peso. Una vez remitidos al Hospital Clínico Veterinario de la Universidad de Murcia, la sintomatología común que presentaban los 26 perros (100\%) era diarrea crónica del intestino delgado, con signos como deposiciones voluminosas, color claro y presencia de esteatorrea. Además, 14 de los perros $(53,84 \%)$ tenían también vómitos crónicos intermitentes, tipo bilioso, generalmente se daban con el estómago vacío. En 8 de los perros $(30,77 \%)$ se observó la presencia de otro signo clínico patente, la pérdida de peso (habían perdido al menos 10\% de su peso habitual desde que empezó la sintomatología), siendo más evidente en aquellos que llevaban más tiempo padeciendo la enfermedad. La sintomatología presentada por los perros de este estudio se consideró crónica al prolongarse durante más de 3 semanas.

\section{PRUEBAS DIAGNÓSTICAS COMPLEMENTARIAS}

A todos los perros se les realizó un análisis bacteriológico y un estudio coprológico de las heces durante 3 días consecutivos a fin de hallar procesos bacterianos o parasitarios que pudieran estar dando lugar a la sintomatología presentada, pero ambas pruebas resultaron negativas. En todos los animales estudiados, los veterinarios de referencia habían prescrito tratamientos antibióticos como metronidazol durante varias semanas, sin éxito total. Se midieron los niveles de TLI en suero sanguíneo, para valorar la funcionalidad pancreática, resultando este parámetro dentro de los límites de referencia $(5-35 \mathrm{ng} / \mathrm{ml})$. Se les realizó un hemograma y un análisis de bioquímica sanguínea para comprobar el estado general de los mismos. Se observó una disminución del hematocrito en seis de los perros $(23,08 \%)$, siendo el límite de referencia $37-55 \%$. 
El valor promedio de todos los casos del estudio fue de $40,08 \%$, con una desviación típica de 7,74. En cuanto a los leucocitos, se encontró leucocitosis en siete de los casos clínicos $(26,92 \%)$, considerándose los límites de referencia $5,5-16,9 \times 10^{3} / \mathrm{mm}^{3}$. El valor promedio de este parámetro fue de $15,04 \times 10^{3} / \mathrm{mm}^{3}$, con una desviación típica de 5,94. En todos los casos se trató de leucocitosis con neutrofilia. Presentaron hipoproteinemia e hipoalbuminemia 12 de los perros de este estudio (46,15\%), siendo leve en seis de ellos (50\%) o moderada en seis de los mismos (50\%), y al hacer el cociente albúmina/globulina se observó que estaba en torno a uno en todos los casos. Se consideró una disminución de ambos parámetros cuando el valor obtenido estaba por debajo del límite inferior de los límites de referencia, siendo el de proteínas totales 5,4-7,7 gr/dl, y el de la albúmina 2,5-3,6 gr/dl. Solo en tres de los casos $(11,54 \%)$ se dio de forma concurrente una disminución del valor hematocrito y de proteínas totales. En lo referente a la fosfatasa alcalina, se observó un aumento en 10 de los perros $(38,46 \%)$, y los límites de referencia estimados fueron 30-120 UI/L, resultando el valor promedio de los perros del estudio de 113,11 UI/L, con una desviación típica de 62,15. El resto de parámetros de bioquímica sanguínea se encontraban dentro del rango de normalidad en todos los perros, resultando el promedio del valor de ALT en 28,26 UI/L (<50 UI/L), con una desviación típica de 14,75; de AST en 25,69 UI/L (<50 UI/L), con una desviación típica de 13,50; de gGT en 3,74 UI/L (1-6,5 $\mathrm{UI} / \mathrm{L})$, con una desviación típica de 1,84; de bilirrubina total en $0,54 \mathrm{mg} / \mathrm{dl}(<1 \mathrm{mg} / \mathrm{dl})$, con una desviación típica de 0,26 ; de creatinina en $0,87 \mathrm{mg} / \mathrm{dl}(0,5-1,5 \mathrm{mg} / \mathrm{dl})$, con una desviación típica de 0,32; y de urea en 44,04 mg/dl (20-50 mg/dl), con una desviación típica de 5,96.

\section{LESIONES MACROSCÓPICAS ENCONTRADAS EN ENDOSCOPIA}

Durante la exploración endoscópica del tracto digestivo anterior se observó que todos los animales presentaban alteraciones digestivas. En ocho de los animales (30,77\%) se observaron lesiones en esófago, 20 de los perros $(76,92 \%)$ presentaban lesiones en el estómago y todos ellos (100\%) tenían lesiones en duodeno. En lo referente a las lesiones del esófago encontradas, eran áreas de aspecto hiperémico e irregular, de tamaño variable. También se valoró el cierre del cardias, y se observó que en 15 de los perros $(57,69 \%)$ cerraba correctamente, siendo incorrecto en 11 de los mismos $(42,31 \%)$. En la exploración gástrica, la mucosa tanto del cuerpo gástrico como del antro pilórico estaba congestiva, engrosada e irregular, y en algún caso con soluciones de continuidad como erosiones y ulceraciones ya fibrosadas. Del total de los animales afectados por lesiones gástricas, el $0 \%$ estaban afectados en grado 0 , el $69,23 \%$ lo estaban en grado 1 , el $30,77 \%$ en grado 2 , y el $0 \%$ en grado 3. En el duodeno, se observó una mucosa hiperémica, congestiva, irregular y friable, ya que al tomar las muestras endoscópicas el tejido se desprendía sin oponer resistencia, y en 20 de los animales (76,92\%) además se vieron soluciones de continuidad como erosiones y úlceras. Las alteraciones en duodeno se consideraron de grado 0 en 0 animales $(0 \%)$, de grado 1 en 20 de los perros $(76,92 \%)$, de grado 2 en 6 de los mismos $(23,08 \%)$, y de grado 3 en 0 casos $(0 \%)$.

\section{LESIONES MICROSCÓPICAS DE LAS MUESTRAS DE BIOPSIA}

Se realizó un estudio histopatológico de las biopsias obtenidas endoscópicamente, utilizando los parámetros publicados por el grupo de estandarización gastrointestinal de la WSAVA (Day y col 2008). En la mucosa gástrica, tomando como valor total al número de animales que sí mostraron afectación gástrica $(76,92 \%)$, la lesión epitelial no estaba presente en 14 de los animales (70\%), leve en tres perros $(15 \%)$ y moderado en tres de los mismos (15\%). La fibrosis estuvo ausente en 16 de los animales (80\%), leve en dos de ellos (10\%) y moderada en dos de los animales (10\%). El infiltrado de linfocitos intraepitelial fue leve en 15 de los casos $(75 \%)$, moderada en tres perros $(15 \%)$ y grave en dos de ellos (10\%). El infiltrado de la lámina propia, compuesto por linfocitos y células plasmáticas de manera difusa, afectó de forma leve a 16 de los perros $(80 \%)$ y grave a cuatro de los animales (20\%). En el intestino delgado, el acortamiento-ensanchamiento de las vellosidades estuvo presente en 24 de los casos $(92,31 \%)$, predominando el grado leve en 15 de los perros $(62,50 \%)$, seguido del grado moderado en nueve de los mismos $(37,50 \%) \mathrm{y}$, por último, se encuentran los animales no afectados, dos de los perros $(7,69 \%)$. En lo referente a la lesión epitelial, 17 de los casos $(65,38 \%)$ no la mostraron, seis de los mismos $(23,08 \%)$ presentaron una lesión leve y tres de los perros $(11,54 \%)$ manifestaron una afectación moderada. La dilatación de vasos linfáticos no estaba presente en 16 de los casos $(61,54 \%)$, en cinco de los animales $(19,23 \%)$ tenían una afectación leve de este parámetro, y en la misma proporción (19,23\%) mostraron una afectación moderada. La fibrosis estaba ausente en 12 de los perros (46,15\%), revelándose en la misma proporción $(46,15 \%)$ en grado leve y en dos de los casos $(7,69 \%)$ en grado moderado. En cuanto al infiltrado de linfocitos intraepiteliales, 14 de los animales mostraron afectación leve $(53,84 \%), 11$ de los mismos tenían una afectación moderada $(42,31 \%)$ y uno de los casos $(3,85 \%)$ evidenciaron una afectación grave. En lámina propia, el infiltrado de linfocitos y células plasmáticas estaba presente en todos los casos $(100 \%)$, presentando un carácter difuso, siendo en 15 de los perros $(57,70 \%)$ de grado moderado y en 11 de los mismos $(42,30 \%)$ se vio una lesión intensa. El infiltrado eosinofílico en lámina propia no estuvo presente en 21 de los animales $(80,77 \%)$, manifestándose en cinco de los casos en grado leve (19,23\%). El infiltrado neutrofílico no se observó en 14 de los perros $(53,84 \%)$, viéndose en 11 de los mismos $(42,31 \%)$ en grado leve y en uno de los casos $(3,85 \%)$ en grado moderado. 


\section{ESTUDIO INMUNOHISTOQUÍMICO}

Todos los perros (100\%) presentaron un inmunofenotipo mixto; en la lámina propia hubo una tinción positiva tanto del marcador CD3 como del marcador CD79, poniendo de manifiesto la presencia de linfocitos $\mathrm{T}$ y de células plasmáticas, de inmunofenotipo B. Ambas poblaciones de células inflamatorias mostraron una afectación difusa dentro de la lámina propia. Los linfocitos intraepiteliales presentaron un inmunofenotipo $\mathrm{T}$, ya que se tiñeron con el marcador CD3. Respecto del marcador MHC II, tres de los casos $(11,54 \%)$ resultaron positivos a su tinción. Con el marcador Ki-67, el que se usó para valorar el índice de proliferación celular entre las células del infiltrado inflamatorio, no se obtuvo un índice de proliferación elevado en ninguno de los perros sometidos al presente estudio.

\section{ANÁLISIS ESTADÍSTICO}

Se llevó a cabo un estudio estadístico para la correlación de los hallazgos histológicos con los datos endoscópicos y bioquímicos, y no se encontró una relación estadística entre el tipo o grado de lesión inflamatoria y las variables de bioquímica clínica consideradas. La disminución del nivel de proteínas totales y la albúmina no presentó una relación estadísticamente significativa con el grado de inflamación de la mucosa gastrointestinal. Los resultados obtenidos entre los estados de hipoalbuminemia y el tipo de infiltrado inflamatorio linfoplasmocitario observados en el estudio histopatológico, a pesar de no ser estadísticamente significativo, sí se aproxima a la significación estadística $(\mathrm{P}<$ $0,1)$. Se encontró una correlación estadística significativa $(\mathrm{P}<0,05)$ entre el tipo de inflamación y la presencia de congestión, hallada endoscópicamente en la mucosa del tracto gastrointestinal anterior, observándose niveles altos de congestión en todos los animales con un infiltrado de tipo linfoplasmocitario. Se obtuvieron resultados que, a pesar de no ser estadísticamente significativo, sí se aproximan a la significación estadística $(\mathrm{P}<0,1)$ entre el grado de congestión endoscópica y la intensidad del infiltrado inflamatorio, ya que una mayor congestión se corresponde con una mayor intensidad del infiltrado linfoplasmocitario, ya sea a nivel gástrico como duodenal. Entre los parámetros de friabilidad e inflamación del duodeno sí hubo una relación estadísticamente significativa $(\mathrm{P}<0,05)$, igual que para los parámetros de esofagitis e incompetencia del cardias.

\section{DISCUSIÓN}

\section{INCIDENCIA DE PRESENTACIÓN DE LA ENFERMEDAD}

La enfermedad inflamatoria crónica intestinal se considera característica de animales de edad media y avanzada (Tams 2003), igual que se considera que hay cierta predisposición genética en algunas razas (Washabau y col 2010). No obstante, como se comprobó en este estudio, se puede manifestar en animales de cualquier edad y raza. En el presente trabajo se encontró un mayor porcentaje de machos que de hembras afectados con la enfermedad, lo que coincide con resultados de otros estudios (Jergens y col 1992; Craven y col 2004), lo que podría sugerir cierta predisposición para este género. No obstante, no existen estudios documentados que comprueben esta predisposición de género (Tams 2003, Hall y German 2007).

\section{SINTOMATOLOGÍA CLÍNICA}

En este estudio todos los animales presentaban diarrea crónica del intestino delgado, en algunos de los casos acompañados de vómitos y pérdida de peso, ello coincide con lo descrito por otros autores, ya que la mayoría de ellos afirma que el vómito y la diarrea se encuentra entre los signos más frecuentes de la enfermedad inflamatoria crónica intestinal, describiendo también como frecuentes la pérdida de peso y las alteraciones del apetito (Jergens y col 1992, Tams 2003, Craven y col 2004).

\section{PRUEBAS DIAGNÓSTICAS COMPLEMENTARIAS}

En el estudio realizado acerca de los datos hematológicos se pudo observar una disminución de los valores de hematocrito en algunos de los casos sometidos a estudio, pero no se produjo de forma generalizada. También se vio leucocitosis con neutrofilia en algunos animales, lo que resulta coherente por la existencia de los fenómenos inflamatorios característicos de esta enfermedad intestinal. Se encuentran similitudes con Hall y German (2007), que señalaron que aunque el examen hematológico a menudo no resulta de ayuda, puede indicar que la anemia puede ser un reflejo de inflamación crónica del tracto gastrointestinal. También se encuentra semejanza con Baez y col (1999), quien realizó un estudio endoscópico en gatos con enfermedad inflamatoria intestinal de estómago e intestino delgado en 32 casos, donde algunos animales presentaron anemia y leucocitosis. Jergens y col (1992) llegaron a las mismas conclusiones en un estudio con 58 perros y 26 gatos. La alteración más frecuente que se encontró en la analítica sanguínea de los perros de este estudio fue la hipoproteinemia, estando la enfermedad inflamatoria crónica intestinal entre las causas más frecuentes de enteropatía con pérdida de proteínas (Tams 2003). Se produce una pérdida de proteínas, tanto albúminas como globulinas, hacia la luz intestinal, manteniéndose el cociente albúmina/globulina en torno a 1 (Rodríguez-Franco 1999). Además, los valores de hipoproteinemia coincidieron en su mayoría con los perros que presentaban pérdida de peso significativa (a partir de 10\%), calculada en función del peso del animal antes de presentar sintomatología y del peso que tenían al llegar al diagnóstico definitivo de la enfermedad. El presente estudio coincide con Hall y German (2007), pues correlacionaron estos datos con un pronóstico más grave. 
Los niveles de fosfatasa alcalina fueron elevados en algunos de los casos. Esto podría ser debido a una alteración hepática secundaria a procesos inflamatorios de naturaleza gastrointestinal, coincidiendo con lo citado por Hall y German (2007). Jergens y col (1992) en su estudio de la EICI aprecian alteraciones bioquímicas similares. No obstante, como se indica en un estudio realizado por Hatayama y col (2001), los niveles elevados de esta enzima pueden tener diversos orígenes, como hueso, especialmente cuando se trata de animales en crecimiento; hígado, en perros con colestasis; inducida por corticoesteroides tanto endógenos como exógenos; o también podrían encontrarse niveles elevados en hembras gestantes. Teniendo en cuenta las edades de los animales de este estudio y los resultados obtenidos en las pruebas diagnósticas, la causa más probable en estos perros es que sea secundaria a alteraciones gastrointestinales.

\section{LESIONES MACROSCÓPICAS ENCONTRADAS EN ENDOSCOPIA}

El $30,77 \%$ de los perros del presente estudio presentaron un esófago con áreas de aspecto hiperémico e irregular, lo que es característico de esofagitis crónica, en este caso, esofagitis por reflujo, ya que se encontraron problemas con el cierre de la válvula del cardias. No obstante, la mayoría de los perros seleccionados para el estudio no mostraban signos de esofagitis, siendo un hallazgo posible pero no habitual de esta enfermedad. A la exploración endoscópica de la porción gástrica, el $76,92 \%$ de los perros presentaron lesiones macroscópicas, y en el $100 \%$ de los casos de este estudio se observaron lesiones macroscópicas de intestino delgado, de mayor o menor intensidad. Las lesiones halladas con una mayor frecuencia, tanto en la sección gástrica como en la duodenal, fueron la presencia de una mucosa congestiva, engrosada, irregular e hiperémica, así como la presencia de soluciones de continuidad fibrosadas, posiblemente se debiera a que un gran número de los perros seleccionados para este estudio tuviesen carácter grave, y friabilidad en el caso de la mucosa intestinal. Estos hallazgos coinciden con los descritos por otros autores, como García-Sancho y col (2007). Baez y col (1999) confirman la friabilidad y engrosamiento de la pared intestinal en 13 de 17 gatos estudiados, y Jergens y col (1992) afirman que las lesiones principales observadas en la EICI son friabilidad, congestión y además eritema, erosión y ulceración de las mucosas del duodeno y estómago indistintamente, siendo semejantes a las encontradas en el presente estudio, en donde los hallazgos endoscópicos mostraron que la friabilidad es un parámetro asociado a inflamación en duodeno. No obstante, la imagen macroscópica puede ofrecer información del proceso, pero es necesario obtener muestras de biopsia para realizar un estudio histopatológico, obligado para confirmar el proceso inflamatorio (Washabau y col 2010).

\section{LESIONES MICROSCÓPICAS DE LAS MUESTRAS DE BIOPSIA}

Se publicaron parámetros estandarizados para la clasificación de la enfermedad inflamatoria crónica intestinal para así poder comparar diferentes estudios de varios autores (Day y col 2008). Sin embargo, incluso con esos estándares sigue existiendo dificultad en llegar a un acuerdo al interpretar biopsias endoscópicas debido a la subjetividad de cada patólogo en la interpretación de las mismas, al procesado y teñido de las muestras, y a la dificultad de aplicar los esquemas de la estandarización. En el presente estudio todos los perros presentaron un infiltrado linfoplasmocitario de moderado a intenso, y de carácter difuso, lo que se considera lo más habitual en EICI (Tams 2003). La presencia de neutrófilos no se suele considerar un hallazgo frecuente en EICI (Tams 2003), aunque en el presente estudio sí se han hallado en algunas de las biopsias debido, probablemente, a una respuesta inflamatoria hacia algún componente microbiano. En cuanto a los eosinófilos, solo se han encontrado en un reducido número de casos, donde es posible que haya intervenido causas alérgicas o parasitarias. Olivero y col (2011) describen en un trabajo bajo los estándares del WSAVA que en EICI la mayoría de los perros presentan linfocitos intraepiteliales en grado leve o moderado, y solo 5\% en grado grave, aproximándose a los resultados del presente estudio. El acortamiento-ensanchamiento de las vellosidades intestinales se ha descrito muy frecuentemente en la EICI, esto coincide con este estudio, ya que este parámetro está presente en la mayoría de los casos. La presencia de dilataciones de los vasos linfáticos, linfagiectasia secundaria al proceso inflamatorio intestinal (ya que por extensión de la inflamación se afecta la red linfática intestinal), son consideradas habituales en la EICI y se han descrito en la mayoría de animales estudiados en otros trabajos (Olivero y col 2011). Sin embargo, debido a que los parámetros estandarizados del WSAVA consideran que los vasos linfáticos deben alcanzar al menos el 50\% de la anchura de la vellosidad intestinal para considerarse linfagiectasias (Day y col 2008), es posible que en el presente estudio sea menor la prevalencia de las mismas. La fibrosis, que afecta a la mitad de los casos de EICI, puede deberse al edema, inflamación y focos pequeños de necrosis, con la consecuente aparición de posterior tejido conjuntivo en las zonas de la mucosa intestinal afectadas (Wilcock y col 1992).

\section{ESTUDIO INMUNOHISTOQUÍMICO}

En el presente trabajo se han realizado técnicas inmunohistoquímicas para la detección de los marcadores de linfocitos CD3 y CD79, que se han utilizado como complemento al diagnóstico histopatológico de los casos de este estudio. En todos los casos se obtuvo una tinción positiva con el marcador CD3, lo que era previsible debido a que los linfocitos intraepiteliales se encuentran 
de forma habitual en el epitelio intestinal, y son de inmunofenotipo T (Kiupel y col 2011). La presencia de linfocitos intraepiteliales en mayor grado del habitual en EICI también se considera frecuente (Washabau y col 2010), probablemente tengan una función de defensa del epitelio frente a organismos patógenos. También existe la posibilidad que estas células puedan migrar desde la lámina propia hacia su localización intraepitelial, como respuesta a una estimulación antigénica (Hall y German 2007). En la lámina propia hubo una tinción positiva a los marcadores CD3 y CD79, presentando un inmunofenotipo mixto, y mostrando una infiltración difusa dentro de la misma, coincidiendo con lo que se suele describir en desórdenes intestinales crónicos en los que se encuadra la EICI (Tams 2003). Los linfocitos B están extensamente representados por células plasmáticas. Los linfocitos $\mathrm{T}$ de la lámina propia son en su mayoría del fenotipo CD4, las cuales reconocen un péptido antigénico representado por las moléculas MHC de clase II (Hall y German 2007). Estos grupos celulares también se encuentran presentes en la EICI lo que coincide con nuestros resultados. Sin embargo, solo en algunos de los casos resultaron positivos a la tinción con el marcador MHC II, probablemente el motivo fuese una mayor reacción del sistema inmune gastrointestinal a los estímulos antigénicos continuos, posiblemente provenientes de la flora microbiana endógena. La expresión de Ki-67 refleja el índice de proliferación celular. Al manifestar las células en división, uno de los objetivos es valorar los posibles casos que presentasen un índice mitótico más elevado, ello podría estar indicando que tendrían características tumorales, pudiendo llegar a ser útil para diferenciar la EICI del linfoma intestinal, ya que en ocasiones los linfomas pueden no ser diagnosticados al utilizar únicamente la histopatología, dando lugar a falsos diagnósticos (Kiupel y col 2011). En el presente estudio en ninguno de los casos se obtuvo un índice de proliferación elevado con el marcador Ki-67.

\section{ANÁLISIS ESTADÍSTICO}

La realización de estudios estadísticos relacionando variables histológicas y clínicas ya ha sido llevada a cabo con anterioridad por otros autores como Baez y col (1999), que correlacionaba los signos clínicos, los hallazgos clinicopatológicos, las pruebas radiográficas y ecográficas, los resultados endoscópicos y los hallazgos histológicos en gatos. Este último trabajo señalaba que los signos clínicos y los hallazgos ecográficos parecen tener una mayor relación con el grado histológico de la EICI en gatos. En el estudio realizado sobre la correlación entre los hallazgos endoscópicos, histológicos y los datos bioquímicos de nuestros casos, se encontró que los animales que tenían un infiltrado de tipo linfoplasmocitario presentaban una tendencia a la pérdida de albúmina, especialmente en los casos de mayor gravedad. En un estudio realizado por Craven y col (2004) en 80 perros con enfermedad inflamatoria intestinal se indicaba que no hay asociación entre el sitio, el tipo y la gravedad de la enfermedad. En medicina veterinaria encontramos estudios que relacionan los bajos niveles de albúmina con un mal pronóstico de la enfermedad. Craven y col (2004) afirman que el grado de hipoalbuminemia tiene relación con el pronóstico de la enfermedad y suele reflejar el grado de lesión histológica. En medicina humana también encontramos estudios que relacionan la hipoalbuminemia con un mal pronóstico en el paciente (Lubrano 2003, Navarro y col 2006, Gisbert y col 2007). Según los resultados obtenidos en el presente estudio, se podría concluir que la disminución de proteínas totales y la albúmina no fue estadísticamente significativa en relación con el grado de inflamación de la mucosa gastrointestinal, no obstante, cualquier patología intestinal que produzca una inflamación, un infiltrado, una congestión o un sangrado suficientes pueden originar una enteropatía perdedora de proteínas, siendo la forma grave de EICI una causa frecuente en perros adultos (Willard 2010). Se observaron niveles altos de congestión vía endoscópica, tanto en estómago como en duodeno, en animales con un infiltrado de tipo linfoplasmocitario. Se debe destacar la gran importancia de este hallazgo, ya que la mayoría de los casos de EICI canina son del tipo linfoplasmocitario, por lo que el grado de congestión podría considerarse como un parámetro válido para sospechar de esta enfermedad. Esto puede deberse a que la mucosa normalmente es tolerante a bacterias endógenas y a los antígenos alimentarios, pero cuando es invadida por un patógeno se produce una reacción inflamatoria en donde las células hospedadoras pueden resultar dañadas, sobre todo si persiste el estímulo antigénico, la lesión patógena continúa o porque hay una anormalidad inherente del sistema inmune. Por lo demás, la disrupción de la barrera mucosa, la desregulación del sistema inmune, las alteraciones en la microflora intestinal o una combinación de estos hechos pueden desestabilizar el sistema y disparar así la inflamación descontrolada (Hall y German 2007), aumentando de esta forma el número de linfocitos y células plasmáticas en la lámina propia. Se desarrolla entonces una inflamación crónica, la que se puede ver reflejada como congestión de la mucosa al realizar la endoscopia. En algunos de los casos de estudio se pudo observar cómo una mayor intensidad del infiltrado estuvo acompañada de una mayor congestión de la mucosa duodenal. Los hallazgos endoscópicos mostraron que la friabilidad está asociada a inflamación en duodeno, ya que la friabilidad suele darse en mucosas irregulares o congestivas (García-Sancho y col 2007), y que los casos en los que no había un correcto cierre del cardias sí había esofagitis por reflujo, muy probablemente debido a que la mala oclusión del esfínter gastroesofágico provocaba la salida de contenido gástrico hacia esófago, dando lugar a los procesos inflamatorios del mismo. 


\section{REFERENCIAS}

Baez JL, MJ Hendrick, LM Walker, RJ Washabau. 1999. Radiographic, ultrasonographic, and endoscopic findings in cats with inflammatory bowel disease of the stomach and small intestine: 33 cases (19901997). J Am Vet Med Assoc 215, 349-354.

Craven M, JW Simpson, AE Ridyard, L Chandler. 2004. Canine inflammatory bowel disease: retrospective analysis of diagnosis and outcome in 80 cases (1995-2002). J Small Anim Pract 45, 336-342.

Day MJ, T Bilzer, J Mansell, B Wilcock, EJ Hall, A Jergens, T Minami, M Willard, R Washabau. 2008. Histopathological standards for the diagnosis of gastrointestinal inflammation in endoscopic biopsy samples from the dog and cat: a report from the world small animal veterinary association gastrointestinal standardization group. J Comp Path 138, 1-43.

García-Sancho M, F Rodríguez-Franco, A Sáinz, C Mancho, A Rodríguez. 2007. Evaluation of clinical, macroscopic, and histopathologic response to treatment in nonhypoproteinemic dogs with lymphocyticplasmacytic enteritis. J Vet Intern Med 21, 11-17.

Gisbert JP, Y González-Lama, J Maté. 2007. 5-Aminosalicylates and renal function in inflammatory bowel disease: a systematic review. .Inflammatory Bowel Diseases 13, 629-638.

Hall EJ, AJ German. 2007. Enfermedades del intestino delgado. En: Ettinger SJ, Feldman EC (ed). Tratado de Medicina Interna Veterinaria. 6 ed. Elsevier Saunders, Madrid, España, Pp 1332-1378.

Hatayama K, Y Nishihara, S Kimura, K Goto, D Nakamura, A Wakita, Y Urasoko. 2001. Serum alkaline phosphatase isoenzymes in laboratory beagle dogs detected by polyacrylamide-gel disk electrophoresis. $J$ Toxicol Sci 36, 653-60.

Hsu SM, L Raine, H Fanger. 1981. Use of avidin-biotin-peroxidase complex $(\mathrm{ABC})$ in immunoperoxidase techniques: A comparasion between $\mathrm{ABC}$ and unlabeled antibody (PAP) procedures. J Histochem Cytochem 29, 577-580.

Jergens AE, FM Moore, JS Haynes, KG Miles. 1992. Idiopathic inflammatory bowel disease in dogs and cats: 84 cases (1987-1990). J Am Vet Med Assoc 201, 1603-1608.
Kiupel M, RC Smedley, C Pfent, Y Xie, Y Xue, AG Wise, JM DeVaul, RK Maes. 2011. Diagnostic algorithm to differentiate lymphoma from inflammation in feline small intestinal biopsy samples. Vet Pathol 48, 212-222.

Lubrano P. 2003. Enfermedad inflamatoria intestinal. Hospital "General José de San Martín", Servicio de Gastroenterología. Residencia de Gastroenterología. Cátedra de Gastroenterología de Postgrado, Faculta de Ciencias Médicas, Universidad Nacional del Litoral, Santa Fe, Argentina.

Navarro-Sanz R, P Juez-Martel, V Sanchis-Bayarri Bernal, E CastellanoVela. 2006. Factores pronósticos en pacientes pluripatológicos de edad avanzada en un hospital de asistencia a crónicos de media y larga estancia (HACMLE). An Med Interna 23, 529-532.

Olivero D, ME Turba, F Gentilini. 2011. Reduced diversity of inmunoglobulin and T-cell receptor gene rearrangements in chronic inflammatory gastrointestinal diseases in dogs. Vet Immunol Immunopathol 144, 337-345.

Rodríguez-Franco F, A Sáinz, MA Tesouro, I Amusategui, O Cortés. 1999. Pharmacological and dietary treatment of canine malabsorption syndrome: A retrospective study of 17 clinical cases. $J$ Vet Med A 46, 439-452.

Simpson JW, RW Else. 1991. Digestive disease in the dog and cat. $1^{\text {st }}$ ed. Blackwell Scientific Publications, Oxford, UK.

Tams TR. 2003. Chronic diseases of the small intestine. In: Tams TR (ed). Handbook of Small Animal Gastroenterology. $2^{\text {nd }}$ ed. W.B.Saunders, Philadelphia, USA, Pp 211-250.

Washabau RJ, MJ Day, MD Willard, EJ Hall, AE Jergens, J Mansell, T Minami, TW Bilzer. 2010. Endoscopic, biopsy, and histopatologic guidelines for the evaluation of gastrointestinal inflammation in companion animals. J Vet Intern Med 24, 10-26.

Wilcock B. 1992. Endoscopic biopsy interpretation in canine or feline enterocolitis. Semin Vet Med Surg (Small Anim) 7, 162-171.

Willard MD. 2010. Trastornos del aparato digestivo. En: Nelson RW, Couto CG (ed). Medicina Interna de pequeños animales. $4^{\mathrm{a}} \mathrm{ed}$ Elsevier Mosby, Barcelona, España, Pp 458-461. 